\title{
Aprendizagem autorregulada da matemática: uma proposta de tutor virtual
}

\author{
Self-regulated learning of mathematics: a virtual tutor proposal
}

\author{
Rosa M. Fontes*, Lia R. Oliveira*, Jorge A. Collus*, Paulo J. Santo*, Rosalina Fernandes*, Carlos P. Semedo** \\ *Universidade do Minho, Portugal, **University of Manchester, UK
}

\begin{abstract}
Resumo
Pretende-se constituir uma nova ferramenta de trabalho/estudo completa — assente na teoria da autorregulação — que permite ao aluno aprender, desenvolver e consolidar os conceitos apresentados na sala de aula, podendo, em tempo real e durante a execução das tarefas, perceber os erros cometidos através da intervenção do tutor virtual que explica e justifica. Este agente digital permitirá observar todo o processo executado pelo aluno, de forma invisível, surgindo apenas quando necessário, de forma lúdica e diversificada. Para verificação e validação deste software abordaremos o domínio de conteúdos GM4 - Geometria e Medidas - e verificaremos as estratégias de resolução de problemas de Polya.

Palavras chave: software educacional para matemática, tutor virtual, autorregulação, matemática, motivação
\end{abstract}

\begin{abstract}
We are developing an online educational software based on self-regulation theory consisting of a virtual tutor and three-dimensional visualizations that will allow the student to learn, develop and consolidate the concepts presented in class, by allowing him to, in real time as the task is being performed, understand his mistakes, through the virtual tutor's intervention. This digital agent follows all the student's steps in the background, appearing only when necessary in diverse and playful ways. In order to validate this software we will look at the domain of GM4 contents - Geometry and Measures - and verify the Polya strategies for problem-solving.
\end{abstract}

Keywords: educational software for mathematics, virtual tutor, self-regulation, mathematics, motivation

Verifica-se um manifesto insucesso na aprendizagem da Matemática, no sistema escolar português (PISA, 2012; TIMSS, 2012). Por outro lado, o número de alunos por computador regista um aumento na ordem de $40 \%$ nos últimos anos (GEPE, 2008). Continuávamos, porém, abaixo de outros países da União Europeia, sendo considerada importante a produção e implementação de conteúdos e aplicações digitais de qualidade (idem, 2008).

Optamos pelo $4^{\circ}$ ano do Ensino Básico visto nesta faixa etária os alunos já manipularem com destreza as ferramentas digitais e apresentarem uma excelente plasticidade cerebral para ultrapassar os desafios da matemática, sendo de extrema importância haver uma assimilação efetiva de conceitos que influenciam, declaradamente, o sucesso académico futuro. O protótipo vai de encontro ao expresso no Diário da República, 1 a $^{\mathrm{a}}$ série - N. ${ }^{\circ}$ 225, no ponto "3.6 - Tutor virtual da Matemática” (Resolução do Conselho de Ministros n. ${ }^{\circ}$ 91/2010 de 19 de Novembro) e é nossa convicção que virá a contribuir para que, socialmente, a matemática deixe de ser vista como um problema.

Para o sucesso do processo de ensino-aprendizagem tem de existir motivação e autorregulação.

Segundo Atkinson (1957), a motivação ou tendência para realizar uma determinada ação (Ta) é o resultado da diferença entre tendência para o sucesso (Ts) e a tendência para o insucesso (Tf).

Para Weiner (1992), a motivação depende de três pares de dimensões: interna/externa, estável/ instável e controlável/incontrolável. As diferentes combinações destas variáveis definiriam a motivação para uma determinada situação.

Covington (1992), refere que a motivação é gerida pela preocupação primária de cada sujeito que é a aceitação própria.

Ryan e Deci (2000), dividem a motivação em extrínseca e intrínseca, sendo a motivação influenciada por questões internas e externas. Esta está intimamente relacionada com a autorregulação e, segundo Rosário (2007), qualquer estudante é capaz de autorregular a sua aprendizagem.

Vygotsky (1978) define autorregulação como capacidade de planificar, organizar e monitorizar o comportamento face a uma determinada situação, tendo este processo na sua base o discurso interno.

Bandura e Zimmerman (1994), propõem a teoria sociocognitiva, na qual a aprendizagem autorregulada resulta dos comportamentos desenvolvidos pelos alunos, sistematicamente orientados para alcançar objetivos que eles próprios definem.

Estudos portugueses (e. g. Machado, Almeida e Silva, 2009; Carvalho, 2013; Cunha, Duarte e Martins, 2010; Caldas, 2011; Santos, 2009; Nobre, 2005) demonstram o benefício dos software como apoio ao ensino-aprendizagem, em particular, da matemática.

Estudos internacionais (e. g. Dagiene e Jasutiene, 2006; Preiner, 2008; Ruthven, Deaney e Hennessy, 2009) corroboram os resultados portugueses.

Após exaustiva incursão em bases de dados bibliográficas (que neste projeto não cabe descriminar), não encontramos qualquer estudo comparável ao que projetamos assim como não encontramos qualquer software com as mesmas características e potencialidades do agora proposto. Realçamos que o protótipo incorpora um agente digital o que implica inteligência artificial e programação de alto nível, já em curso como mostraremos adiante.

Comprovados os benefícios dos software utilizados em contexto de ensino da matemática, como esperado, questionaremos a temática das máquinas de ensinar, 
ideia que vem sendo explorada desde o século XIX, particularmente no século XX, por autores como Pressey (anos 20) ou Skinner (anos 50) e, mais tarde, recuperada por Benjamin (1988) e Moeglin (1993) entre outros, discussão essa que ressurge, atualmente, com grande enfoque no Canadá, em França e nos EUA.

Esta investigação tem como intenção combater o insucesso escolar na disciplina de Matemática. Para o efeito, propomo-nos criar um software educativo inovador com visualização tridimensional $\mathrm{e}$ incorporando um tutor virtual (simulando um tutor humano mas com características de invisibilidade, de acompanhamento contínuo e de registo de acções). Sublinhamos que não é nossa pretensão substituir o professor, bem pelo contrário, este desempenha um papel fulcral em todo o processo sendo a vertente pedagógica da sua inteira responsabilidade. Simultaneamente pretendemos, de forma pragmática, rentabilizar os recursos atualmente existentes nas escolas (computadores).

O tutor virtual apresenta um sistema dinâmico envolvendo linguagem escrita (enunciados), sonora (comentários de feedback) e visual (visualização tridimensional de imagens geométricas, interativas). O som e a escrita estão sempre presentes nas explicações prévias da temática a ser abordada. Propõe um conjunto de exercícios para verificação e consolidação da aprendizagem (estratégia de ensino habitual em Matemática). Apresenta, como inovação criativa de maior destaque, uma função autorreguladora do erro, visto que os alunos erram, com frequência, quer na interpretação dos enunciados, quer na realização das tarefas propostas. Ou seja, no ecrã do computador, através do browser (o sistema funciona online) o software apresenta um balão a identificar o erro, respetiva explicação e correção. Aqui entra o som, que poderá, eventualmente, ser opcional (questão a estudar até pelas questões de acessibilidade que estão previstas). Os alunos terão, assim, a oportunidade de se autorregularem à medida que verificam os seus erros e compreendem o porquê dos mesmos.

Em simultâneo, o professor terá acesso às tarefas executadas pelo aluno e, ao erro cometido por cada um individualmente: o software terá incorporada uma base de dados, onde cada aluno é identificado por uma ficha individual à qual o professor terá acesso através de uma senha. Esta ficha terá um histórico dos erros cometidos por cada aluno.

Realçamos que o nosso agente digital opera em qualquer browser e em qualquer máquina, a saber: computadores, tablets, telemóveis com acesso à internet entre outros.

São nossos objectivos: melhorar os resultados dos alunos no domínio de conteúdo GM4; promover a autorregulação nos alunos; motivar os alunos com um software novo e desafiante; fornecer ao professor informação individualizada sobre as dificuldades e formas de resolução dos problemas de cada aluno; usar o tutor virtual na sala de aula durante a lecionação do domínio de conteúdo GM4, permitindo ao aluno e ao professor a verificação das dificuldades da apreensão/compreensão; refletir sobre a pertinência/ contributo do uso de máquinas de ensinar (Cf. Benjamin, 1988; Moeglin, 1993) nos nossos dias.

Em síntese, a nossa proposta de Tutor Digital apresenta as seguintes vantagens: não apresenta custos adicionais para as instituições escolares, nem para alunos nem para professores; rentabiliza os recursos informáticos existentes no parque escolar; pode ser usado em qualquer máquina ligada à Internet; e fornece visualização tridimensional de figuras geométricas.

\section{Método}

Esta investigação insere-se num plano do tipo misto, uma vez que envolve a recolha de dados quantitativos (e.g. número de erros, número de alunos) e qualitativos (e.g. a variável que contempla a satisfação ou não com a utilização do software...). Apresenta uma natureza e um modelo experimental, uma vez que uma variável independente (software) é manipulada com o intuito de se estudar os seus efeitos na variável dependente (resultados dos alunos a GM4).

Pretendendo-se desenvolver um produto de raiz - o software em questão - analisando a situação inicial, o problema com que nos defrontamos (o insucesso a Matemática), construindo um quadro teórico de análise, constituindo um estado da arte, procedendo à realização de vários sub-estudos implicados no processo de design de um artefacto tecnológico desta natureza (entre eles a avaliação da usabilidade e a validação do valor do protótipo finalizado e operacional), adoptamos a Metodologia do Desenvolvimento (Richey e Nelson,1996; Van Der Maren, 1996; De Ketele e Roegiers, 1999; Van den Akker,1999; Giardina, 1999; Oliveira, 2004) como quadro orientador de todo o estudo.

Para efeitos de validação (para além da obrigatória avaliação da usabilidade), incidiremos no tema GM4 (geometria e medidas) lecionado no $4^{\circ}$ ano do $1^{\circ}$ ciclo do Ensino Básico.

Este sistema encontra-se já prototipado numa fase ainda incipiente mas já ilustrativa do sistema a criar na sua forma final.

\section{Participantes}

Os participantes serão alunos de 10 turmas (5 de cada sistema) do $4^{\circ}$ ano do Ensino Básico, bem como os seus professores. Serão asseguradas todas as orientações e obrigações éticas. Relativamente à análise dos dados, efetuaremos análise de conteúdo (Bardin, 1991). A amostra é não probabilística e por conveniência, uma vez que garantimos acesso a turmas com diferentes caraterísticas e de diferentes sistemas (público e privado).

\section{Instrumentos}

Os instrumentos utilizados na realização deste estudo serão o software em questão, um ebook informativo disponibilizado antes da aplicação do software e os questionários online, pós aplicação para avaliação da satisfação/insatisfação relativamente à utilização do software. 


\section{Procedimentos}

A metodologia do desenvolvimento utilizada neste estudo será estruturada em duas fases: 1) Constituição da amostra, aplicação e validação/avaliação do software; 2) Recolha de dados complementares e análise global. A primeira fase consistirá na realização de seis tarefas: a) obtenção de autorização das respetivas direções de escola, professores e consentimento informado dos pais dos alunos; b) acesso ao historial académico dos alunos participantes, no domínio de conteúdo GM4; c) esclarecimento aos professores e alunos sobre o funcionamento do software e das suas funcionalidades com disponibilização de um ebook; formação prévia através da realização de um pré-teste no software; d) aplicação em sala de aula do nosso software, durante a lecionação da matéria referente ao domínio de conteúdo GM4; e) realização do teste de avaliação (integrado no software) relativo à matéria referente ao domínio de conteúdo GM4; f) realização de inquéritos por questionários online acerca da satisfação/insatisfação, motivação gerada pelo software, opinião geral e melhoramentos possíveis relativos ao uso do software educativo, a aplicar a alunos e professores. A segunda fase consistirá na realização de duas tarefas: a) análise dos dados obtidos através do software, relativamente à proficiência, número de erros, quais os erros, entre outras informações; b) reflexão global sobre a pertinência de uso de máquinas de ensinar nos nossos dias.

\section{Resultados Expectáveis}

No que concerne aos resultados esperados prevemos: promoção da autorregulação dos alunos; sucesso escolar dos alunos no domínio de conteúdos GM4; aumento da motivação dos alunos para a aprendizagem da matemática; aumento da proficiência dos alunos.

\section{Referências}

Atkinson, J. W. (1957). Motivational dererminants of risk-taking behavror. Psythological Retien, 64, 359-372.

Bardin, L. (1991). Análise de Conteúdo. Lisboa: Edições. Benjamin, L. T. Jr. (1988). A history of teaching machines. American Psychologist, 43(9), 703712.

Caldas, M. C. S. (2011). A Integração Curricular das TIC: Estudo de Caso tomando como exemplo a Geometria no Ensino Básico. (Dissertação de mestrado). Universidade do Minho.

Carvalho, L. T. N. (2013). Ambiente Virtual de Aprendizagem Matemática em contexto educativo. Dissertação de mestrado. Lisboa: Instituto de Educação da Universidade de Lisboa.

Covington, M. V. (1992). Making the grade: A self-worth perspective on motivation and school reform. Cambridge: Cambridge University Press.

Cunha, B., Duarte, E. \& Martins, J. (2010). A Matemática com as TIC no processo de ensino-aprendizagem:- Construção de uma unidade didáctica. Pós-Graduação. Porto:
Escola Superior de Educação de Paula Frassinetti.

Dagiene, V. \& Jasutiene, E. (2006). Developing Dynamic Sketches for Teaching Mathematics in Basic Schools. Acedido 20 de junho, 2014, em http://ims.mii.lt/valentina/publ/Dagiene_Ja sutiene.pdf

De Ketele, J-M. \& Roegiers, X. (1999). Metodologia de Recolha de Dados. Fundamentos dos métodos de observações, de questionários, de entrevistas $e$ de estudo de documentos. Lisboa: Instituto Piaget.

Diário da República, 1. ${ }^{a}$ série - N. 225 - 19 de Novembro de 2010, Acedido 7 de julho, 2013, em http://dre.pt/pdf1s/2010/11/22500/0528805 307.pdf

GEPE. (2008). Modernização tecnológica do ensino em Portugal. Estudo de diagnóstico. Lisboa: Editorial Ministério da Educação.

Giardina, M. (1999). L'interactivité, le multimédia et l'apprentissage. Paris: L’Harmattan.

Machado, J., Almeida, L., Silva, B. (2009). Ensinoaprendizagem da matemática com recurso a software educativo: Atitudes e rendimento académico dos alunos, Innovación Educativa, 19, 41-50.

Moeglin, P. (1993). Le paradigme de la machine, à enseigner. Études de communication, 14, 91-103.

Nobre, M. (2005). O uso do software Matlab para o estudo de alguns tópicos de álgebra linear. Acedido 20 de maio, 2014, em http://www.ucb.br/sites/100/103/TCC/2200 5/MarcelloNobreCardoso.pdf

Oliveira, L. R. (2004). A comunicação educativa em ambientes virtuais: um modelo de design de dispositivos para o ensino-aprendizagem na Universidade. Braga: Centro de Investigação em Educação.

Preiner, J. (2008). Introducing Dynamic Mathematics Software to Mathematics Teachers: the Case of GeoGebra. Dissertation in Mathematics Education. Salzburg: Faculty of Natural Sciences University of Salzburg.

Polya, G. (1957). How to Solve It. $2^{\mathrm{a}}$ ed. Princeton: Princeton University Press.

Richey, R. C. E Nelson, W. A. (1996), Developmantal Research. In D. H. Jonassen (ed.), Handbook of Research for Educational Communications and Technology: A Project of the Association for Educational Communications and Technology (1213-1245). New York: Simon \& Schuster Macmillan.

Rosário, P., Mourão, R., Nuñez, J. C., González-Pienda, J., Solano, P. \& Valle, A. (2007). Eficacia de un programa instruccional para la mejora de processos y estrategias de aprendizaje en la enseñanza superior. Psicothema, 19(3), 422-427.

Ryan, R. \& Deci, E. L. (2000). Intrinsic and extrinsic motivations. Classic definitions and new 
directions. Contemporary Educacional Psychology, 25, 54-67.

Ruthven, K., Deaney, R. \& Hennessy, S. (2009). Using Graphing Software to Teach about Algebraic Forms: A Study of Technology-Supported Practice in Secondary-School Mathematics. Educational Studies in Mathematics, 71(3), 279-297. Acedido 14 de janeiro, 2014, em https://www.educ.cam.ac.uk/people/staff/ruthv en/RuthvenESMpreprint.pdf

Santos, N. B.(2009). O estudo das funções matemáticas polinomiais elementares com o uso do software Graph. Monografia apresentada ao Setor de Pós- Graduação da Universidade do Extremo Sul Catarinense - UNESC, para a obtenção do título de especialista em Educação Matemática.

Skinner, B. F. (1953). Science and human behavior. New York: Macmillan.

TIMSS 2011 Desempenho em Matemática. (2012). Acedido 10 de março, 2014, em http://www.portugal.gov.pt/media/793501/TIM SS\%202011\%20MATH\%204.pdf
Van den Akker, J. (1999). Principles and Methods of Development Research. In J. van den Akker; R. Branch, R. et al. (eds.) Design Approaches and Tools in Education and Training (1-15). Netherlands: Kluwer Academic Publisher.

Van Der Maren, J. M. (1996). Méthodes de Recherche pour l'Education. 2 ${ }^{\mathrm{a}}$ ed. Bruxeles: DeBoeck Université.

Vygotsky, L. S. (1978). Mind in society: the development of higher psychological processes. Cambridge: Harvard University Press.

Weiner, B. (1992). Human motivation: Metaphors, theories and research. Newbury Park, CA: SAGE Publications.

Zimmerman, B. J. \& Bandura, A. (1994). Impact of Self-Regualtory Influences on Writing Course Attainment. American Educational Research Journal, 31(4), 845-862. 